\title{
Protein Array-Based Detection of Proteins in Kidney Tissues from Patients with Membranous Nephropathy
}

\author{
Shuqiang Wang, Yang Lu, Quan Hong, Xiaodong Geng, Xu Wang, Wei Zheng, \\ Chengcheng Song, Chunling Liu, Meng Fan, Yue Xi, Mandi Guo, and Di Wu
}

Department of Nephrology, PLA General Hospital, Institute of Nephrology, Beijing Key Laboratory of Kidney Disease, State Key Laboratory of Kidney Diseases, National Clinical Research Center for Kidney Diseases, Beijing 100853, China

Correspondence should be addressed to DiWu; wudi@301hospital.com.cn

Received 19 November 2016; Revised 25 January 2017; Accepted 29 January 2017; Published 27 February 2017

Academic Editor: Gang Liu

Copyright (c) 2017 Shuqiang Wang et al. This is an open access article distributed under the Creative Commons Attribution License, which permits unrestricted use, distribution, and reproduction in any medium, provided the original work is properly cited.

\begin{abstract}
Membranous nephropathy (MN) is an autoimmune inflammatory disease in which proteins related with plenty of biological processes play an important role. However, the role of these proteins in the pathogenesis of MN is still unclear. This study aimed to screen differential proteins in kidney tissue samples from MN patients by using protein arrays and determine the pathways involved in the pathogenesis of MN. This study first tested a quantitative protein array (QAH-INF-3) and two semiquantitative protein arrays (L-493 and L-507) with normal renal tissue and identified L-493 as the most appropriate assay to compare protein levels between MN tissues and normal control tissues. The L-493 array identified 66 differentially expressed proteins (DEPs) that may be associated with MN. The gene oncology (GO) and protein-protein interaction (PPI) analyses revealed several processes potentially involved in $\mathrm{MN}$, including extracellular matrix disassembly and organization, cell adhesion, cell-cell signaling, cellular protein metabolic process, and immune response $(P<0.05)$. We suggest that these different pathways work together via protein signaling and result in the pathogenesis and progression of $\mathrm{MN}$.
\end{abstract}

\section{Introduction}

Membranous nephropathy (MN) is an autoimmune inflammatory disease and the primary cause of nephrotic syndrome in adults, contributing to $20 \%-40 \%$ of end stage renal disease (ESRD) cases [1]. The characteristic pathological manifestation of MN is thickening of the glomerular basement membrane (GBM), which originates from glomerular visceral epithelial cells and endothelial cells. This thickening is due to deposition of immune complex IgG and C3 under the podocytes. However, the pathways involved in the process of GBM thickening are still unclear.

According to the previous study, autoantibodies and complements play important roles in the development and progression of MN [2]. In 2009, Beck Jr. et al. identified the anti-M-type phospholipase A2 receptor (anti-PLA2R) as an autoantibody target in approximately $70 \%$ of patients with idiopathic MN [3]. The detection of anti-PLA2R antibodies has been used clinically. The role of the membrane attack complex of the complement has been confirmed [4-6].
Proteins, which act as the executor of cell function, may take part in the whole pathogenetic process of MN. The present study concentrated on several families of cytokines such as the interleukin family, tumor necrosis factor (TNF) family, and transforming growth factor (TGF) family; nonetheless, large-scale screening and function analysis of kidney proteins are rare [7-13].

Protein microarrays are advanced and efficient technology to study proteins involved in kidney disease systematically because the arrays have the potential to screen thousands of proteins in a high-throughput manner with a smaller sample as compared with mass spectrometry (MS) and other traditional methods such as western blot and enzyme-linked immunosorbent assays (ELISAs) [14, 15]. According to the working principle, protein arrays can be classified as direct detection and indirect detection. The direct detection method could detect the proteins captured directly. The indirect detection method detects the proteins with antibody and markers such as biotin and fluorescein. In our study, we compared two different indirect methods, including 
the quantitative array Quantibody ${ }^{\circledR}$ Human Inflammation Array 3 (QAH-INF-3) and two semiquantitative L-series antibody arrays L-507 and L-493 with the goal of determining a suitable protein array to detect proteins in kidney tissue and perform a large-scale screen for $\mathrm{MN}$-related proteins and pathways.

\section{Materials and Methods}

2.1. Sample Collection. Our study was approved by the institutional research ethics committee of the Chinese People's Liberation Army General Hospital (No. S2015-061-01). All procedures performed in this study involving human participants were in accordance with the ethical standards of the institutional and national research committee and with either the 1964 Declaration of Helsinki and its later amendments or the comparable ethical standards. All of the samples were obtained from the Nephrology Department of the Chinese People's Liberation Army General Hospital. A total of 15 kidney samples harvested via percutaneous renal biopsy and nephrectomy were included in the study as a discovery set: six samples from idiopathic MN patients and nine normal samples from renal cell carcinoma (RCC) patients. All of the MN patients are IMN diagnosed explicitly by renal biopsy pathology with exceptions for other diseases such as autoimmune diseases, infection, diabetes, and hypertension. There are 25, 28, and 32 glomeruli in each quality control specimen, respectively.

2.2. Protein Extraction. After the fresh samples were collected, the impurities and blood were washed out with PBS immediately. The process of centrifugation and removal of the supernatant was repeated for the samples until the tissue became nearly colorless. The tissue was dissociated and lysed according to the manufacturer's instructions. After the lysates were centrifuged, the protein concentrations in the supernatant were detected by using BCA methods (BCA Protein Assay Kit, Pierce, Prod \#: 23227, France). The samples were diluted in sample buffer to the desired concentration in a volume of $100 \mu \mathrm{L}$.

2.3. Protein Microarray Detection. We chose the three following protein arrays to compare their performance in assessing the protein levels in kidney tissue: the quantitative protein chip QAH-INF-3 (RayBiotech, Inc., Norcross, GA) and the semiquantitative protein L-series antibody array chips L-507 and L-493 (RayBiotech, Inc., Norcross, GA). QAH-INF-3 is a type of multiplexed sandwich ELISA-based quantitative array platform that enables researchers to accurately and simultaneously detect the concentration of 40 proteins by building a standard curve with a serial dilution of standard samples. L-507 and L-493 could separately detect 507 and 493 proteins based on biotin labeling and laser fluorescence scanning techniques, respectively. For detection, $12.5 \mu \mathrm{g}$ and $6.25 \mu \mathrm{g}$ samples from a single tissue were used for QAH-INF3 , and $50 \mu \mathrm{g}$ and $25 \mu \mathrm{g}$ from the same kidney sample were used for L-507 and L-493.

Protein hybridization was conducted with the QAHINF-3, L-507, and L-493 arrays according to the respective manufacturer's instructions. The fluorescent images were scanned by an Axon GenePix (Molecular Devices, Inc., Orleans, USA), and the data were analyzed with GenePix Pro 6.0 software (Molecular Devices, Inc., Orleans, USA).

Hybridization proceeded according to the manufacture of QAH-INF-3, L-507, and L-493. The signal fluorescence images were scanned by Axon GenePix (Molecular Devices, Inc., Orleans, USA). The data was analyzed with software GenePix Pro 6.0 (Molecular Devices, Inc., Orleans, USA).

2.4. ELISA Verification. To verify the results, EpCAM-1, CTSD (Abcam, Inc., Cambridge, UK), and ADAMTS-4 (R\&D Systems, Inc., Minnesota, USA) were selected as confirmation markers and tissue lysates of $\mathrm{MN}$ and $\mathrm{N}$ were measured by ELISA kits. The procedures were carried out following the specification of the kits.

2.5. Immunohistochemistry Verification. To avoid the false overexpression of transmembrane proteins caused by tissue lysates, we performed immunohistochemistry of $\mathrm{MN}$ and normal control with CTSD and CD46. Immunohistochemistry was carried out following the conventional methods of fixing tissues, paraffin-embedding, serial section $(3 \mathrm{~mm})$, antigen retrieval, blocking endogenous peroxidase activity, adding antibodies, incubation, and microscopic examination. The antibodies of CTSD and CD46 are both mouse monoclonal antibody (Santa Cruz Biotec, Inc., USA).

2.6. Statistical Analyses. The protein array performances among QAH-INF-3, L-507, and L-493 were assessed by the fold change of protein expression between the two concentrations of the loading samples. We defined proteins with a ratio between 1.3 and $3.0(12.5 \mu \mathrm{g}$ versus $6.25 \mu \mathrm{g}$ group in QAH-INF-3, $50 \mu \mathrm{g}$ versus $25 \mu \mathrm{g}$ group in L-507 and L-493; the theoretical ratio was 2.0) as measurable proteins in kidney tissue and counted the number of available proteins on each chip to assess the performance of protein chips.

Student's $t$-test ( $t$-test) was used to address the significance of the quantitative data. Proteins with a fold change of $\mathrm{MN} / \mathrm{N}>1.4$ and a $P$ value $<0.05$ were considered to be differentially expressed proteins (DEPs). The Database for Annotation, Visualization and Integrated Discovery v6.8 Beta (DAVID v6.8 Beta, https://david-d.ncifcrf.gov) was used for gene oncology (GO) analysis. The Search Tool for the Retrieval of Interacting Genes/Proteins v10 (STRING v10, http://www.string-db.org/) was applied for protein-protein interaction analysis.

\section{Results and Discussion}

3.1. Results. The general clinical characteristics of $\mathrm{MN}$ patients and normal control subjects are shown in Table 1 . The gender ratios are the same (female: male $=2: 1$ ), and there was no significant difference regarding age, serum creatinine (Scr), and estimate glomerular filtration rate $(P>0.05)$ while the urine protein and albumin (Alb) showed significant differences $(P<0.05)$. The sample selection was consistent with the clinical situations of MN patients and normal control subjects. 
TABLE 1: Clinical characteristics of MN versus NC.

\begin{tabular}{lccc}
\hline & $\begin{array}{c}\text { Membranous } \\
\text { nephropathy } \\
(\text { mean } \pm \text { SD) }\end{array}$ & $\begin{array}{c}\text { Normal control } \\
(\text { mean } \pm \text { SD) }\end{array}$ & $P$ value \\
\hline $\begin{array}{l}\text { Age } \\
\text { Urine }\end{array}$ & $28.33 \pm 8.50$ & $35.33 \pm 5.51$ & $P>0.05$ \\
protein $(\mathrm{mg} / \mathrm{dL})$ & $500.00 \pm 100.00$ & $16.67 \pm 15.28$ & $P<0.05$ \\
$\begin{array}{l}\text { Scr }(\mathrm{umol} / \mathrm{L}) \\
\text { Alb }(\mathrm{g} / \mathrm{L})\end{array}$ & $59.90 \pm 16.07$ & $71.27 \pm 15.45$ & $P>0.05$ \\
$\mathrm{eGFR}$ & $30.30 \pm 3.19$ & $43.13 \pm 2.51$ & $P<0.05$ \\
$\mathrm{~mL} /\left(\mathrm{min} \cdot 1.73 \mathrm{~m}^{2}\right)$ & $159.33 \pm 23.76$ & $119.53 \pm 12.07$ & $P>0.05$ \\
\hline
\end{tabular}

3.1.1. L-493 Shows a Good Performance for Kidney Samples Compared to QAH-INF-3 and L-507. We first compared the performance of the three arrays in detecting proteins in normal kidney samples.

For QAH-INF-3, the fluorescence image, standard curves, and scatter plot are shown in Supplementary Figure 1 in Supplementary Material available online at https://doi.org/ $10.1155 / 2017 / 7843584$. All 40 proteins could be detected in QAH-INF-3 and the exact signal intensities analysis showed that only $22.5 \%(9 / 40)$ of the signal intensity ratios of $12.5 \mu \mathrm{g} /$ $6.25 \mu \mathrm{g}$ were between 1.3 and 2.0. Obviously, QAH-INF-9 does not meet our requirements.

For L-507, the fluorescence image and the signal values are shown as Supplementary Figure 2. The average ratio of the $50 \mu \mathrm{g} / 25 \mu \mathrm{g}$ signal intensities was 0.99 , which was far less than the theoretical ratio of 2.0. The exact signal intensities ratio of $50 \mu \mathrm{g} / 25 \mu \mathrm{g}$ showed that $26(5.13 \%)$ proteins were not detected, merely $61(12.03 \%)$ were between 1.3 and 3.0, 411 $(91.07 \%)$ were less than 1.3 , and $9(1.78 \%)$ were larger than 3. The majority of the ratios are biased to the lower value.

For L-493, the fluorescence image and scatter plot are shown in Figure 1. The average ratio of the $50 \mu \mathrm{g} / 25 \mu \mathrm{g}$ signal intensities was 2.27 , which is close to the theoretical ratio of 2.0. The exact signal intensity ratios of the $50 \mu \mathrm{g} / 25 \mu \mathrm{g}$ results showed that $4(0.81 \%)$ proteins were not detected, 214 (43.41\%) were between 1.3 and 3.0 (Figure 1(c)), 184 (37.32\%) were between 0 and 1.3 , and only $9(18.46 \%)$ were larger than 3.

The primary analysis showed that L-493 could detect more available proteins $(214,43.41 \%)$ compared to QAHINF-3 $(9,22.5 \%)$ and L-507 (61, 12.03\%), suggesting that L493 is more suitable for detecting proteins in kidney tissue. Therefore, we selected L-493 to detect DEPs in tissues from $\mathrm{MN}$ patients and normal controls.

3.1.2. DEPs in MN Detected by L-493 Play a Crucial Role for MN Progression via Regulation of a Molecular Network. The fluorescence images of $\mathrm{MN}$ and normal control were shown in Figure 2. L-493 identified 66 DEPs (fold change of $\mathrm{MN} / \mathrm{N}>1.4, P<0.05$ ), and they were all upregulated in $\mathrm{MN}$ compared to normal tissue (Figure 2(g)). The exact data was in Supplementary Table 1. GO analysis (Figure 3(a)) found several significant biological processes associated with these proteins, including extracellular matrix disassembly and organization, cell adhesion, cell-cell signaling, cellular protein metabolic process, and the immune response $(P<$ 0.05).

More importantly, we constructed a molecular network based on protein-protein interactions (PPI) by using STRING (Figure 3(b)). The network and GO analysis (Table 3) showed that proteins such as EpCAM, FER, SYK, CDH1, KRT8, KRT19, and KRT18 are associated with cell adhesion; hormones such as POMC, IAPP, PTHLH, and ACE are associated with cell metabolism; CXCL5, CCR7, CD55, CD46, and CD47 are associated with immune activation; and TF, FN1, ADAMTS4, APCS, CTSD, and MMP13 are associated with extracellular matrix remodeling. What is more, this entire functional zone is interconnected by mutual proteins.

3.1.3. Verification Results of ELISA and Immunohistochemistry. As shown in Table 2 and Figure 4, the ratio of $\mathrm{MN} / \mathrm{N}$ in EpCAM, CTSD, and ADAMTS4 is 1.44, 2.16, and 1.77 in ELISA $(P<0.05)$, similar to $1.75,1.91$, and 2.06 in L493, respectively. And immunohistochemistry also showed the overexpression of CTSD and CD46 in MN (Figure 5). CTSD is widely overexpressed in tubulointerstitium and slightly in glomerulus (Figure 5(d)), while CD46 has a partly enhanced impression in tubulointerstitium (Figure 5(c)). These confirmed our results of protein microarray L- 493 .

3.2. Discussion. In this study, we explored the use of different protein chips for testing kidney tissues and performed a largescale screen of proteins in kidney tissues from MN patients to elucidate the mechanism of the pathophysiological process.

We first tested three different protein chips. It was found that $\mathrm{L}-493$ was most suitable for detection proteins in kidney tissue. Compared with the QAH-INF-3 and L-507 chips, we deduced that the proteins examined by L- 493 were abundant in kidney tissue and that most of these proteins could be detected. Moreover, the different qualities of the antibodies in the protein chip were also important. Next, we examined the DEPs by using the L- 493 chips.

We found that proteins such as CXCL5, CCR7, CD55, and CD97 were associated with the immune response. It was reported that CXCL5 and CCR7 were involved in immunocyte activation and inflammation after bacteria and virus infection $[16,17]$. CD55 is the decay accelerating factor for complement; it is involved in the regulation of the complement cascade. CD97 takes part in the cell-cell signal transferring in immune activation. $\mathrm{MN}$ has high relevance with infection and immunity; autoantibody and proteins take the essential role in it [18]. Immune activation acts as the initiative factor in the physiopathology of $\mathrm{MN}$. Borza suggested that immune response may act directly on podocytes and endothelial cells to secrete extracellular matrix and thicken the GBM [5].

GBM disassembly and reorganization are the final pathological changes of MN. We identified proteins such as MMP13, FN1, CTSD, and ADAMTS4, which are associated with GBM disassembly and reorganization. For instance, MMP13 is the member of the matrix metalloproteinase family that contributes to the breakdown of extracellular matrix in tissue remodeling, and previous studies have shown that this family may be associated with collagen-induced arthritis and 


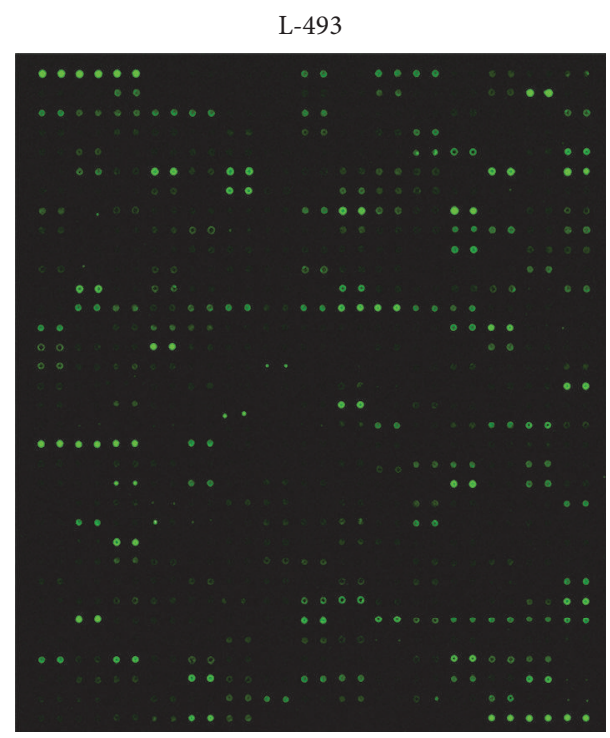

(a)

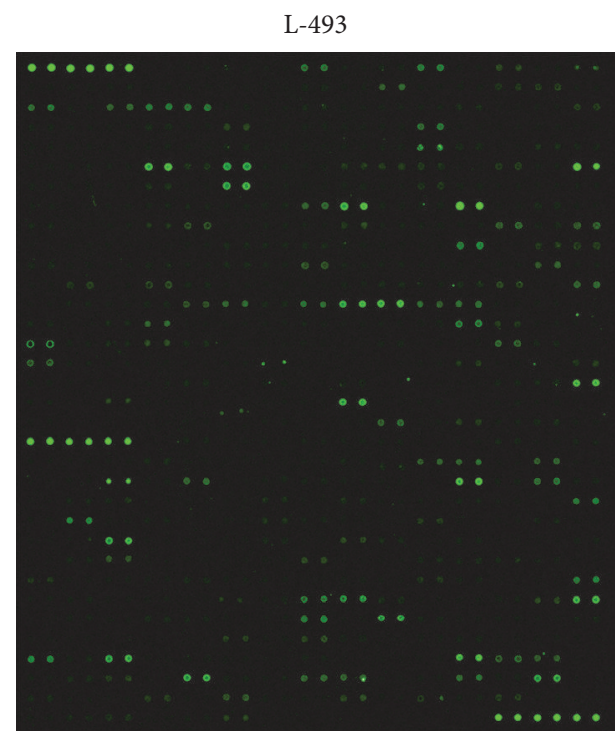

(b)

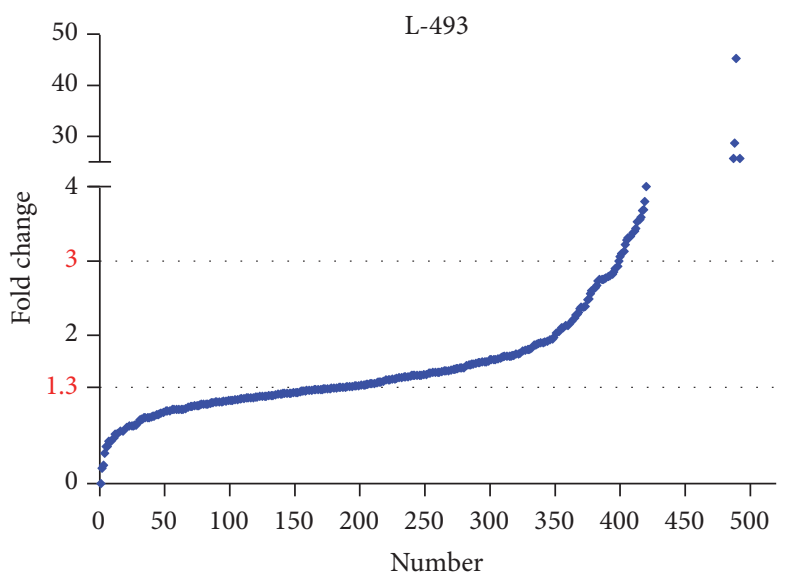

(c)

FIGURE 1: Fluorescence images and scatter plot of the semiquantitative protein array L-493 (493 proteins) in detecting proteins in kidney tissue. (a) and (b) are fluorescence images of $50 \mu \mathrm{g}$ and $25 \mu \mathrm{g}$ of the sample, respectively. (c) represents the scatter plot of L-493. There are 214 proteins with a fold change between 1.3 and 3.0.

TABLE 2: Results of ELISA verification.

\begin{tabular}{lcccc}
\hline Protein & MN & N & Fold change & $P$ value \\
\hline EpCAM & $277.20 \pm 35.32 \mathrm{pg} / \mathrm{mL}$ & $192.85 \pm 29.59 \mathrm{pg} / \mathrm{mL}$ & 1.44 & $<0.05$ \\
CTSD & $73.07 \pm 16.75 \mathrm{ng} / \mathrm{mL}$ & $33.76 \pm 8.04 \mathrm{ng} / \mathrm{mL}$ & 2.16 & $<0.05$ \\
ADAMST-4 & $728.50 \pm 163.75 \mathrm{pg} / \mathrm{mL}$ & $411.50 \pm 112.96 \mathrm{pg} / \mathrm{mL}$ & 1.77 & $<0.05$ \\
\hline
\end{tabular}

glioblastoma [19-22]. Both arthritis and glioblastoma involve matrix disassembly in a similar manner as MN. We suggested that MMP-13 may play the same role in MN. Fibronectin 1 (FN1) may be a major component of the excessive accumulation of extracellular matrix (ECM) in the kidney glomeruli. Deltas et al. reported that FN1 is associated with thin basement membrane nephropathy (TBMN) [23]. Herbach et al. and Alvarez et al. reported increased glomerular expression of FN1 in mice and humans with diabetic nephropathy [24, 25]. Cathepsin D (CTSD) is a protein with the activity of proteolysis; it is mostly overexpressed in tubulointerstitium and the GO analysis in our research found it important in ECM remodeling. This has been supported by several previous researches about chronic kidney disease [26, 27]. We suggested that CTSD may enroll in the interstitial fibrosis after transdifferentiation of renal tubular epithelial cells induced by heavy proteinuria in $\mathrm{MN}$.

Proteins such as CDH1, EpCAM, and the KRT family may be involved in cell adhesion in MN. According to previous studies, cell adhesion plays a role in glomerulonephritis, but 


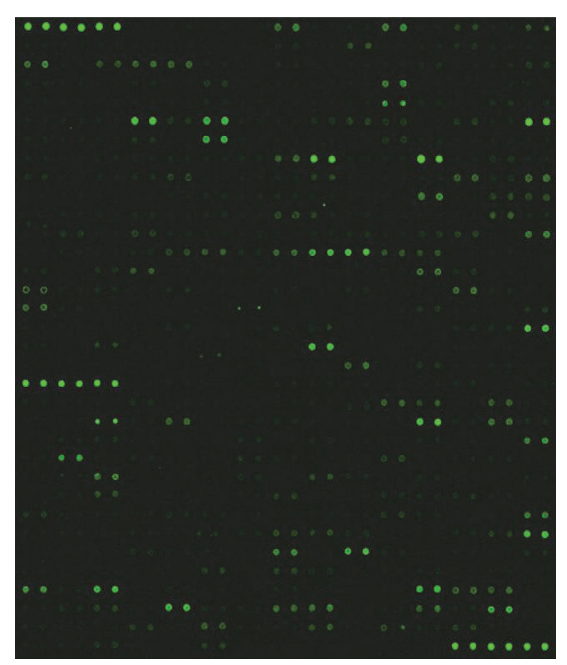

(a)

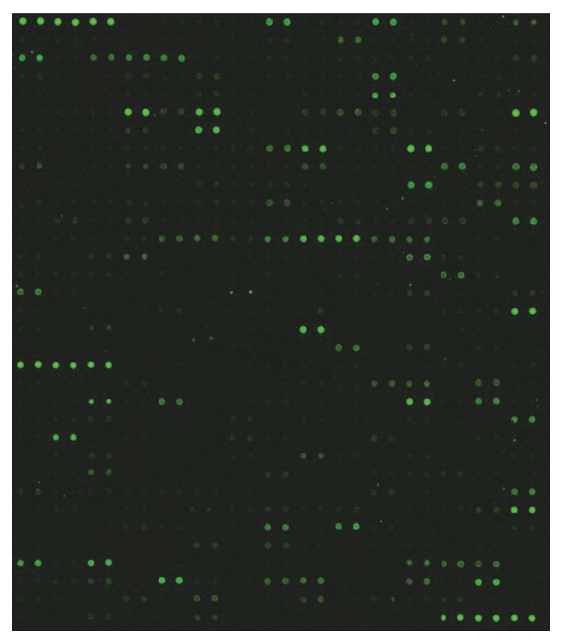

(d)

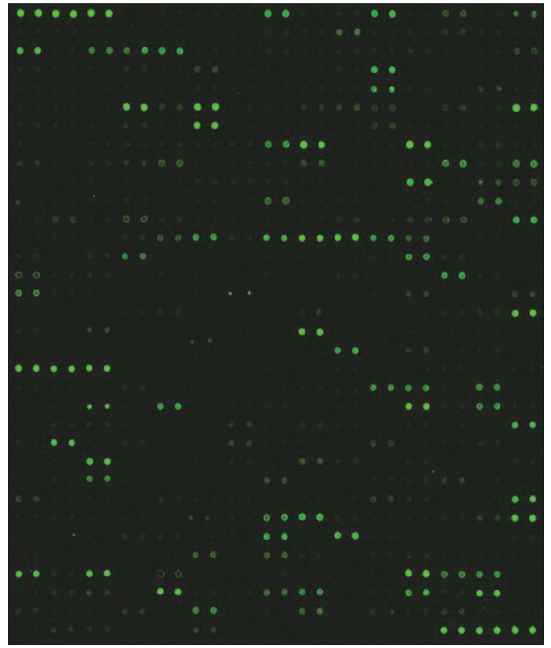

(b)

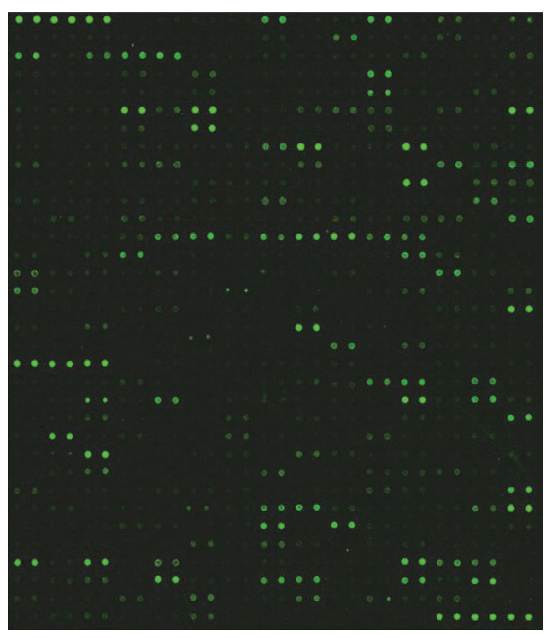

(e)

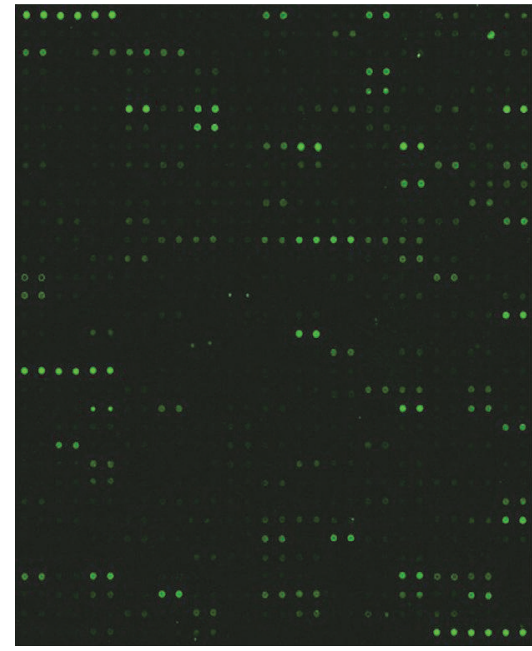

(c)

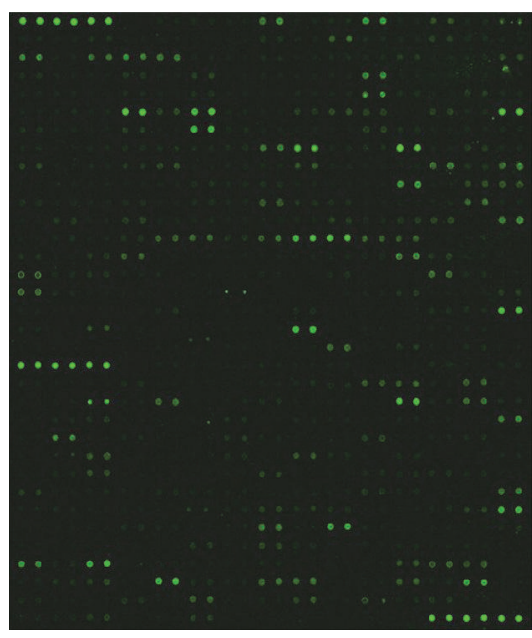

(f)

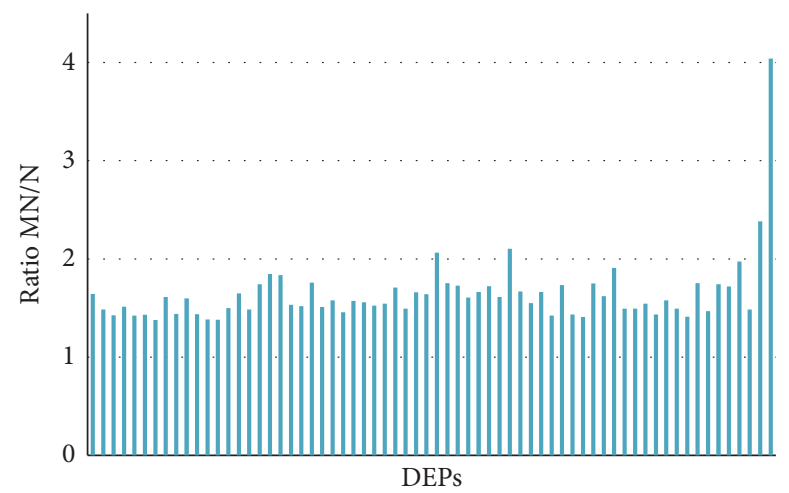

(g)

FIGURE 2: Fluorescence images and histogram of L-493 in MN versus N. (a), (b), and (c) are fluorescence images of 3 cases in normal control group; (d), (e), and (f) are 3 cases in MN. (g) is the histogram showing the ratio of MN/N in 66 upregulated different expression proteins (DEPs). 


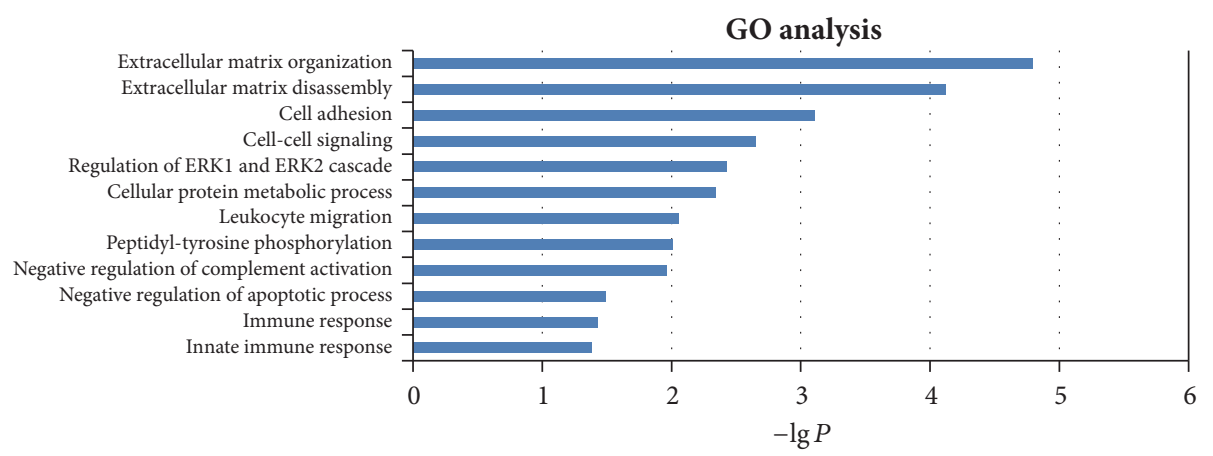

(a)

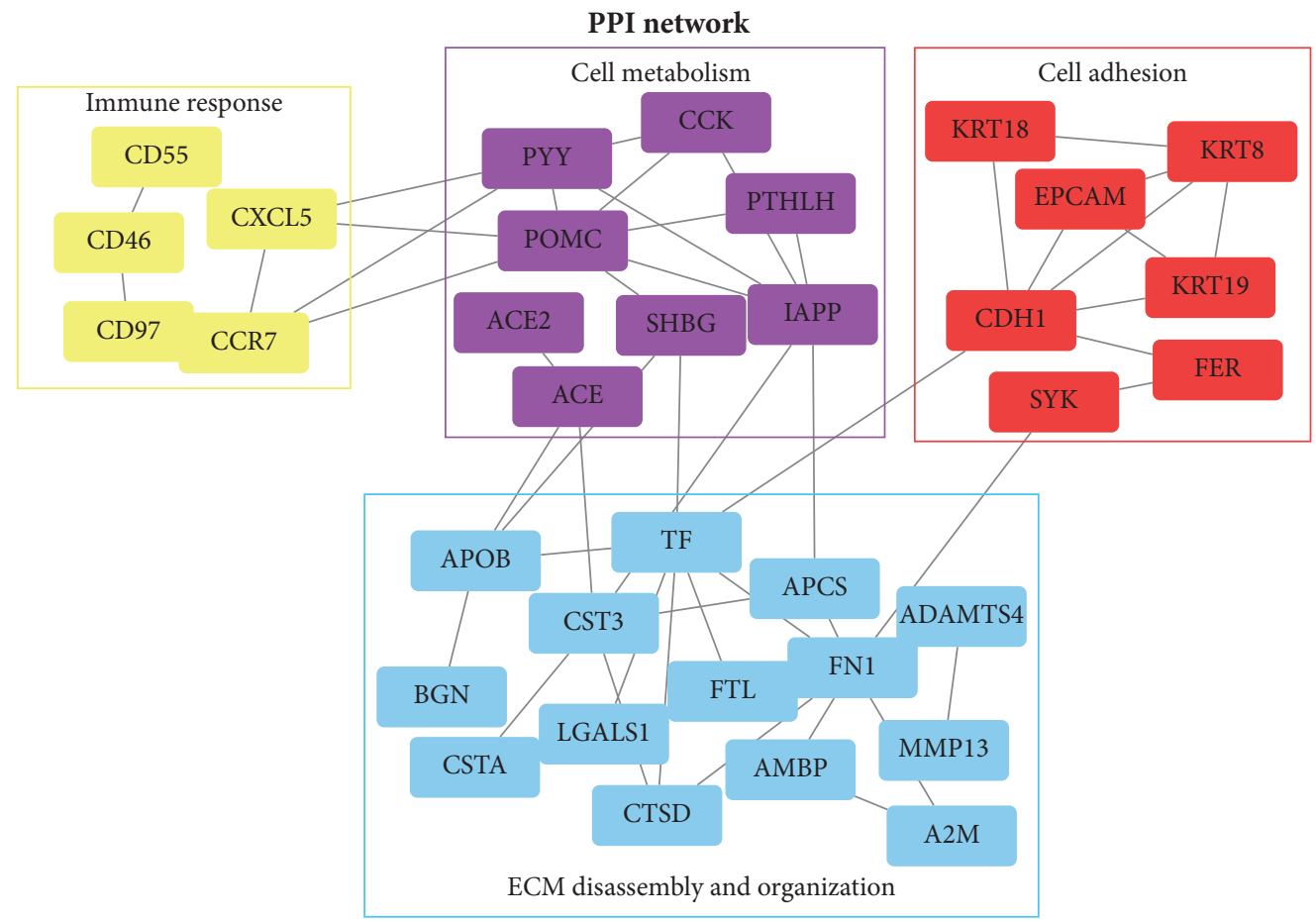

(b)

FIGURE 3: Gene oncology and predicted protein-protein interaction network of the differentially expressed proteins (DEPs). (a) shows the first 12 GO pathways $(P<0.05)$ of DEPs; the $y$-axis represents $-\lg P$. (b) shows the PPI network of main proteins, with different colors representing different functional zones: yellow, immune response; purple, cell metabolism; red, cell adhesion; and blue, extracellular matrix disassembly and organization.
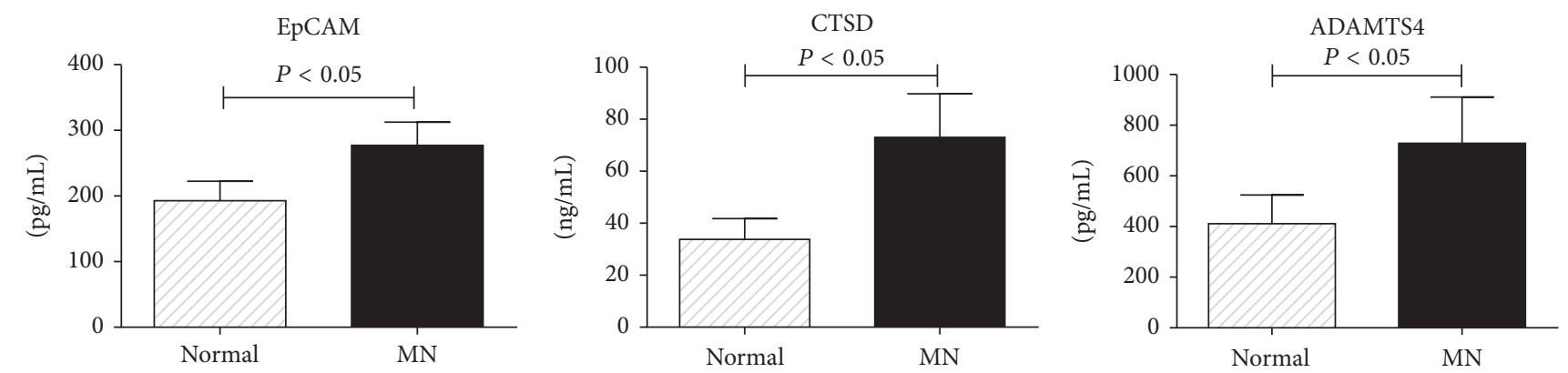

FIgURE 4: ELISA verification of EpCAM, CTSD, and ADAMTS4. The $y$-axis represents the sample concentrations. The levels of these proteins from $\mathrm{MN}$ tissue were higher than those in normal control tissue $(P<0.05)$. 
TABLE 3: GO pathways of differentially expressed proteins.

\begin{tabular}{|c|c|c|}
\hline Term & $P$ value & Genes \\
\hline Extracellular matrix organization & $1.6 \times 10^{-5}$ & FN1, ADAMTS4, CDH1, BGN, CTSD, MMP13, IBSP, A2M, CD47 \\
\hline Extracellular matrix disassembly & $7.6 \times 10^{-5}$ & FN1, ADAMTS4, CDH1, CTSD, MMP13, A2M \\
\hline Cell adhesion & $7.9 \times 10^{-4}$ & FN1, CNTN2, APC, FER, EPHA3, IBSP, CD97, CD47 \\
\hline Cell-cell signaling & $2.3 \times 10^{-3}$ & PYY, PTHLH, IAPP, CXCL5, CD97, POMC \\
\hline Regulation of ERK1 and ERK2 cascade & $3.7 \times 10^{-3}$ & EPHB1, FN1, SYK \\
\hline Cellular protein metabolic process & $4.5 \times 10^{-3}$ & BIRC5, B2M, IAPP, MMP13, ACE2, CD55, APCS, ACE, CST3, POMC \\
\hline Leukocyte migration & $8.8 \times 10^{-3}$ & FN1, APOB, CD244, CD47 \\
\hline Peptidyl-tyrosine phosphorylation & $9.9 \times 10^{-3}$ & EPHB1, FER, ROR1, EPHA3 \\
\hline Negative regulation of complement activation & $1.1 \times 10^{-2}$ & CD46, CD55 \\
\hline Negative regulation of apoptotic process & $3.3 \times 10^{-2}$ & BIRC5, FN1, ANGPTL4, KRT18, BNIP2, ACSTD1 \\
\hline Immune response & $3.8 \times 10^{-2}$ & TNFSF13B, DEFB1, CCR7, CXCL5, CD97 \\
\hline Innate immune response & $4.1 \times 10^{-2}$ & B2M, DEFB1, SYK, CD244, FER, CD46, CD55, APCS \\
\hline
\end{tabular}

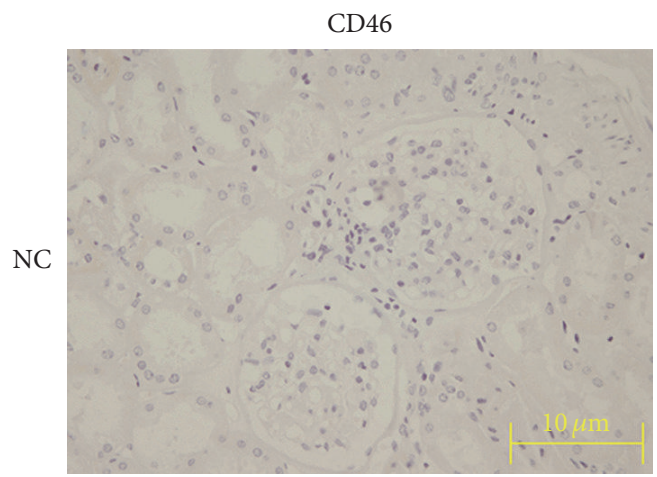

(a)

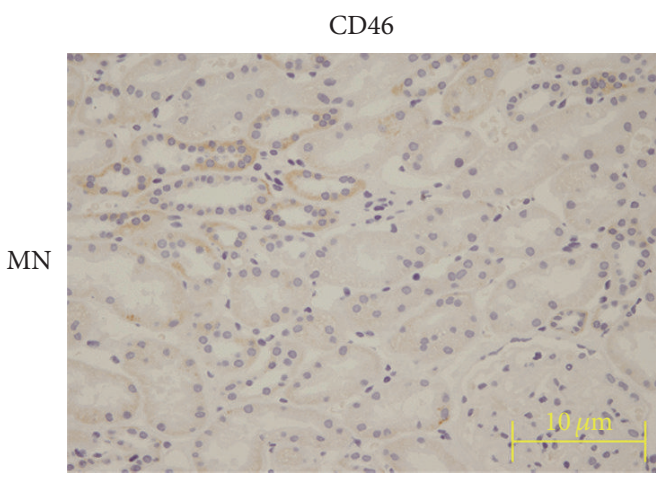

(c)

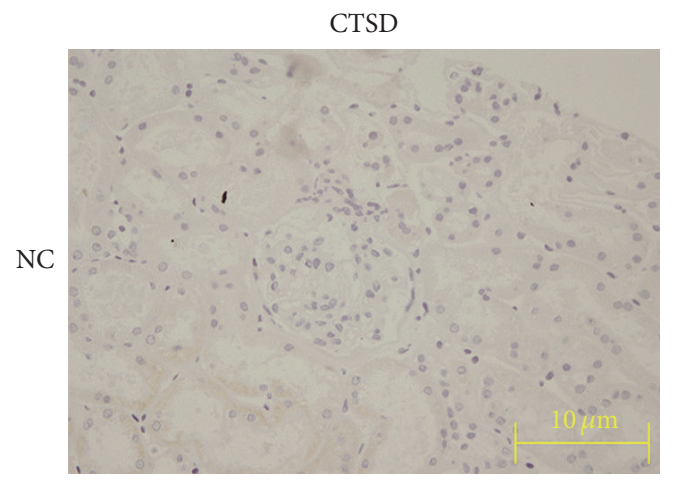

(b)

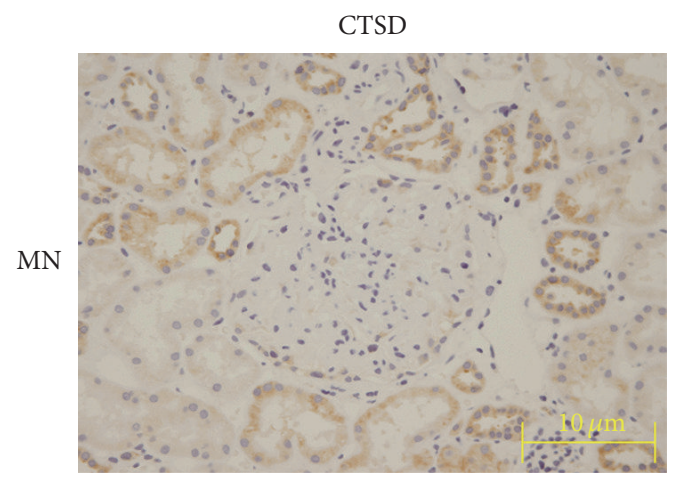

(d)

FIGURE 5: Immunohistochemistry verification of CD46 and CTSD. The images are at 400x magnification. The expressions of CD46 (a) and CTSD (b) in normal control tissues are almost negative. CD46 is partly overexpressed in tubulointerstitium of MN (c); CTSD is widely positive expressed in tubulointerstitium and slightly positive in glomerulus of MN (d).

its mechanism is still under investigation [28, 29]. Zheng et al. suggested that $\mathrm{CDH} 1$ contributes to the development of diabetic nephropathy (DN) [30]. Because the main pathological change during the early stages of $\mathrm{DN}$ is the thickening of the GBM and ECM expansion (similar to MN), we deduced that cell adhesion plays a role in $\mathrm{MN}$.

Interestingly, in our results, hormones such as POMC, PTHLH, CCK, and IAPP are associated with cell metabolism in MN. We checked the GENECARDS database and found that all of these proteins are expressed in kidney. Previous studies found that PTH affects podocyte function [31], but the exact mechanism of how hormones affect GBM is unclear. We hypothesized that hormones changed the metabolism of podocytes and endothelial cells to promote the thickening of the GBM.

More importantly, we built a molecular network to elucidate the mechanism of MN. Within this network, immune activation, cell adhesion, cell-cell signal transduction, and cell 
metabolism could act on the ECM and promote the reorganization of the GBM. Although how these pathways interact is still unclear, we found several proteins that may serve as the link among them. For instance, spleen tyrosine kinase (SYK), as an important component of the intracellular signaling pathway for various immunoreceptors, could also regulate cell adhesion [32]. Serotransferrin (TF) is always upregulated during serious infection but may stimulate cell proliferation and matrix remodeling as well [33]. Alpha-2-macroglobulin $(\mathrm{A} 2 \mathrm{M})$ is a protease inhibitor and protein transporter, but $\mathrm{GO}$ analysis suggests its involvement in ECM organization and cell adhesion. Therefore, we believe that different pathways interact with each other and concomitantly contribute to the pathogenesis and progression of MN.

It should be mentioned that our study has some limitations. First, the protein microarray has some deficiencies, of which the most important one is that it cannot distinguish the protein location from glomeruli and tubules in kidney. Second, acquiring samples was really challenging, and high cost of the protein chip limited the sample size in the experiments and validation. In addition, we were also unable to compare multiple disease groups such as $\mathrm{MN}$ with $\operatorname{IgA}$ nephropathy, minimal change disease (MCD), and focal segmental glomerular sclerosis (FSGS). More studies are needed to address this in the future.

\section{Conclusion}

In conclusion, we used the protein array to identify differential proteins expressed in kidney tissue from MN patients, screened out proteins that may be related with $\mathrm{MN}$, and reported several possible pathways that could be involved in ECM accumulation. This study is a preliminary exploration, the differential expressed proteins and pathways supply us with more targets to study $\mathrm{MN}$, and we hope to continue pursuing this discovery with more thorough research.

\section{Competing Interests}

The authors declare that there is no conflict of interests regarding the publication of this paper.

\section{Acknowledgments}

This work was supported by the National Basic Research Development Program of China (2014CBA02005) and the National Natural Sciences Foundation of China (no. 81470949 , no. 81370785 , no. 81330019 , and no. 61671479).

\section{References}

[1] K. Dahan, "Membranous nephropathy: diagnosis, new insights in pathophysiology, and therapeutic approach," La Revue de Médecine Interne, vol. 37, no. 10, pp. 674-679, 2016.

[2] S. Bally, H. Debiec, D. Ponard et al., "Phospholipase A2 receptor-related membranous nephropathy and mannan-binding lectin deficiency," Journal of the American Society of Nephrology, vol. 27, no. 12, pp. 3539-3544, 2016.
[3] L. H. Beck Jr., R. G. B. Bonegio, G. Lambeau et al., "M-type phospholipase A2 receptor as target antigen in idiopathic membranous nephropathy," New England Journal of Medicine, vol. 361, no. 1, pp. 11-21, 2009.

[4] D.-B. Borza, "Alternative pathway dysregulation and the conundrum of complement activation by IgG4 immune complexes in membranous nephropathy," Frontiers in Immunology, vol. 7, article no. 157, 2016.

[5] D. Borza, "Glomerular basement membrane heparan sulfate in health and disease: a regulator of local complement activation," Matrix Biology, 2016.

[6] R. A. Sinico, N. Mezzina, B. Trezzi, G. M. Ghiggeri, and A. Radice, "Immunology of membranous nephropathy: from animal models to humans," Clinical and Experimental Immunology, vol. 183, no. 2, pp. 157-165, 2016.

[7] H. S. Lee, "Mechanisms and consequences of TGF- $\beta$ overexpression by podocytes in progressive podocyte disease," Cell and Tissue Research, vol. 347, no. 1, pp. 129-140, 2012.

[8] H. S. Lee, "Pathogenic role of TGF- $\beta$ in the progression of podocyte diseases," Histology and Histopathology, vol. 26, no. 1, pp. 107-116, 2011.

[9] M. Nangaku, S. J. Shankland, and W. G. Couser, "Cellular response to injury in membranous nephropathy," Journal of the American Society of Nephrology, vol. 16, no. 5, pp. 1195-1204, 2005.

[10] B. Sutariya, D. Jhonsa, and M. N. Saraf, “TGF- $\beta$ : the connecting link between nephropathy and fibrosis," Immunopharmacology and Immunotoxicology, vol. 38, no. 1, pp. 39-49, 2016.

[11] C. Bantis, P. J. Heering, S. Aker et al., "Tumor necrosis factor- $\alpha$ gene G-308A polymorphism is a risk factor for the development of membranous glomerulonephritis," American Journal of Nephrology, vol. 26, no. 1, pp. 12-15, 2006.

[12] D. Kalavrizioti, M. Gerolymos, M. Rodi et al., "T helper (Th)cytokines in the urine of patients with primary glomerulonephritis treated with immunosuppressive drugs: can they predict outcome?" Cytokine, vol. 76, no. 2, pp. 260-269, 2015.

[13] D. Thibaudin, L. Thibaudin, P. Berthoux et al., “TNFA2 and d2 alleles of the tumor necrosis factor alpha gene polymorphism are associated with onset/occurrence of idiopathic membranous nephropathy," Kidney International, vol. 71, no. 5, pp. 431437, 2007.

[14] M. Valentina, F. Jan, N. L. Peder, Z. Bo, D. Hongjie, and K. Pernille, "Cytokine detection and simultaneous assessment of rheumatoid factor interference in human serum and synovial fluid using high-sensitivity protein arrays on plasmonic gold chips," BMC Biotechnology, vol. 15, no. 1, article no. 73, 2015.

[15] P. V. Hauser, P. Perco, I. Mühlberger et al., "Microarray and bioinformatics analysis of gene expression in experimental membranous nephropathy," Nephron-Experimental Nephrology, vol. 112, no. 2, pp. e43-e58, 2009.

[16] H. Lee, Y.-T. Ahn, J.-H. Lee, C.-S. Huh, and D.-H. Kim, "Evaluation of anti-colitic effect of lactic acid bacteria in mice by cDNA microarray analysis," Inflammation, vol. 32, no. 6, pp. 379-386, 2009.

[17] K. M. Sepuru, B. Nagarajan, U. R. Desai, and K. Rajarathnam, "Molecular basis of chemokine CXCL5-glycosaminoglycan interactions," Journal of Biological Chemistry, vol. 291, no. 39, pp. 20539-20550, 2016.

[18] W. L. Lai, T. H. Yeh, P. M. Chen et al., "Membranous nephropathy: a review on the pathogenesis, diagnosis, and treatment," Journal of the Formosan Medical Association, vol. 114, no. 2, pp. 102-111, 2015. 
[19] Y. Sakamaki, H. Sasamura, K. Hayashi et al., "Absence of gelatinase (MMP-9) or collagenase (MMP-13) attenuates adriamycin-Induced albuminuria and glomerulosclerosis," NephronExperimental Nephrology, vol. 115, no. 2, pp. e22-e32, 2010.

[20] S. E. Campbell, A. Sood, D. J. Argyle, L. Nasir, S. A. Argyle, and D. Bennett, "The cloning and functional analysis of canine matrix metalloproteinase-13 gene promoter," Gene, vol. 286, no. 2, pp. 233-240, 2002.

[21] C. Wang, X. Tong, X. Jiang, and F. Yang, "Effect of matrix metalloproteinase-mediated matrix degradation on glioblastoma cell behavior in 3D PEG-based hydrogels," Journal of Biomedical Materials Research Part A, vol. 105, no. 3, pp. 770-778, 2017.

[22] E. Ha and K. C. Mun, "Effects of cyclosporine on metalloproteinase in endothelial cells," Transplantation Proceedings, vol. 44, no. 4, pp. 991-992, 2012.

[23] C. Deltas, A. Pierides, and K. Voskarides, "Molecular genetics of familial hematuric diseases," Nephrology Dialysis Transplantation, vol. 28, no. 12, pp. 2946-2960, 2013.

[24] N. Herbach, I. Schairer, A. Blutke et al., "Diabetic kidney lesions of GIPRdn transgenic mice: podocyte hypertrophy and thickening of the GBM precede glomerular hypertrophy and glomerulosclerosis," American Journal of Physiology-Renal Physiology, vol. 296, no. 4, pp. F819-F829, 2009.

[25] M. L. Alvarez, M. Khosroheidari, E. Eddy, and J. Kiefer, "Role of microRNA 1207-5P and its host gene, the long non-coding RNA Pvtl, as mediators of extracellular matrix accumulation in the kidney: implications for diabetic nephropathy," PLoS ONE, vol. 8, no. 10, Article ID e77468, 2013.

[26] T. Yurube, T. Takada, T. Suzuki et al., "Rat tail static compression model mimics extracellular matrix metabolic imbalances of matrix metalloproteinases, aggrecanases, and tissue inhibitors of metalloproteinases in intervertebral disc degeneration," Arthritis Research and Therapy, vol. 14, no. 2, article no. R51, 2012.

[27] C. Fox, P. Cocchiaro, F. Oakley et al., "Inhibition of lysosomal protease cathepsin $\mathrm{D}$ reduces renal fibrosis in murine chronic kidney disease," Scientific Reports, vol. 6, Article ID 20101, 2016.

[28] A. B. Singh and R. C. Harris, "Epidermal growth factor receptor activation differentially regulates claudin expression and enhances transepithelial resistance in Madin-Darby canine kidney cells," The Journal of Biological Chemistry, vol. 279, no. 5, pp. 3543-3552, 2004.

[29] A. Škoberne, A. Behnert, B. Teng et al., "Serum with phospholipase A2 receptor autoantibodies interferes with podocyte adhesion to collagen," European Journal of Clinical Investigation, vol. 44, no. 8, pp. 753-765, 2014.

[30] M. Zheng, L.-L. Lv, Y.-H. Cao et al., "A pilot trial assessing urinary gene expression profiling with an mRNA array for diabetic nephropathy," PLoS ONE, vol. 7, no. 5, Article ID e34824, 2012.

[31] E. Ritz, M.-L. Gross, and R. Dikow, "Role of calcium-phosphorous disorders in the progression of renal failure," Kidney International. Supplement, no. 99, pp. S66-S70, 2005.

[32] S. P. McAdoo, G. Bhangal, T. Page, H. T. Cook, C. D. Pusey, and F. W. K. Tam, "Correlation of disease activity in proliferative glomerulonephritis with glomerular spleen tyrosine kinase expression," Kidney International, vol. 88, no. 1, pp. 52-60, 2015.

[33] H. Tamouza, J. M. Chemouny, L. Raskova Kafkova et al., “The IgA1 immune complex-mediated activation of the MAPK/ERK kinase pathway in mesangial cells is associated with glomerular damage in IgA nephropathy," Kidney International, vol. 82, no. 12, pp. 1284-1296, 2012. 

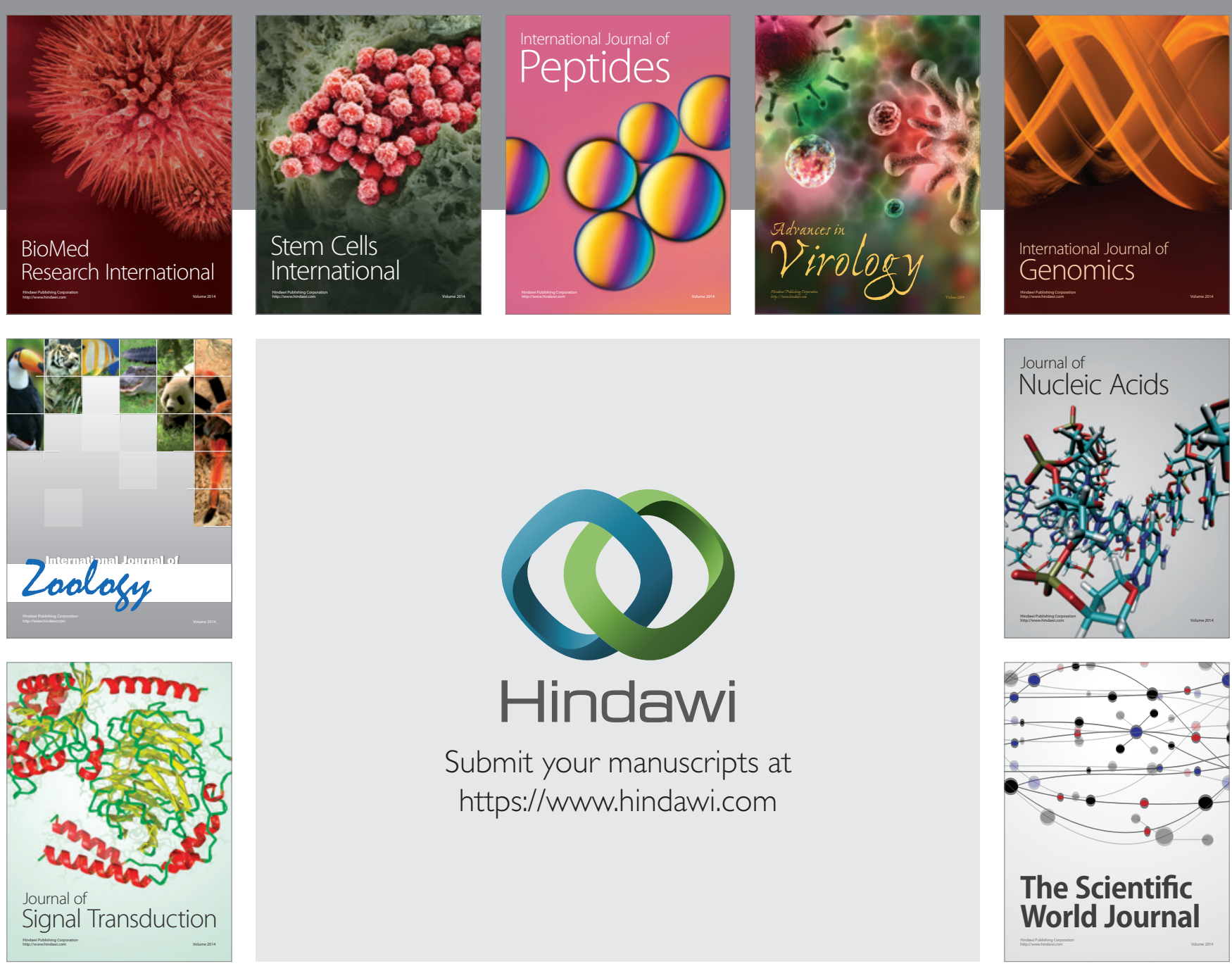

Submit your manuscripts at

https://www.hindawi.com
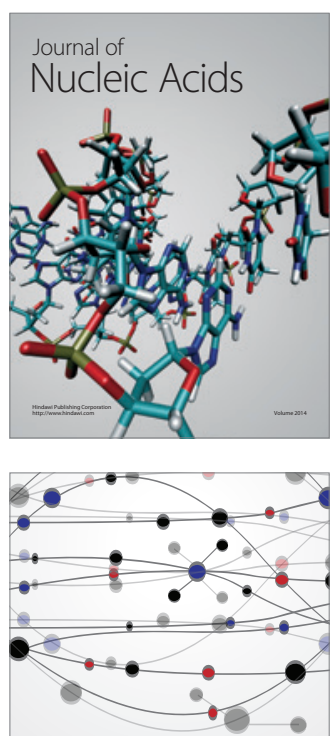

The Scientific World Journal
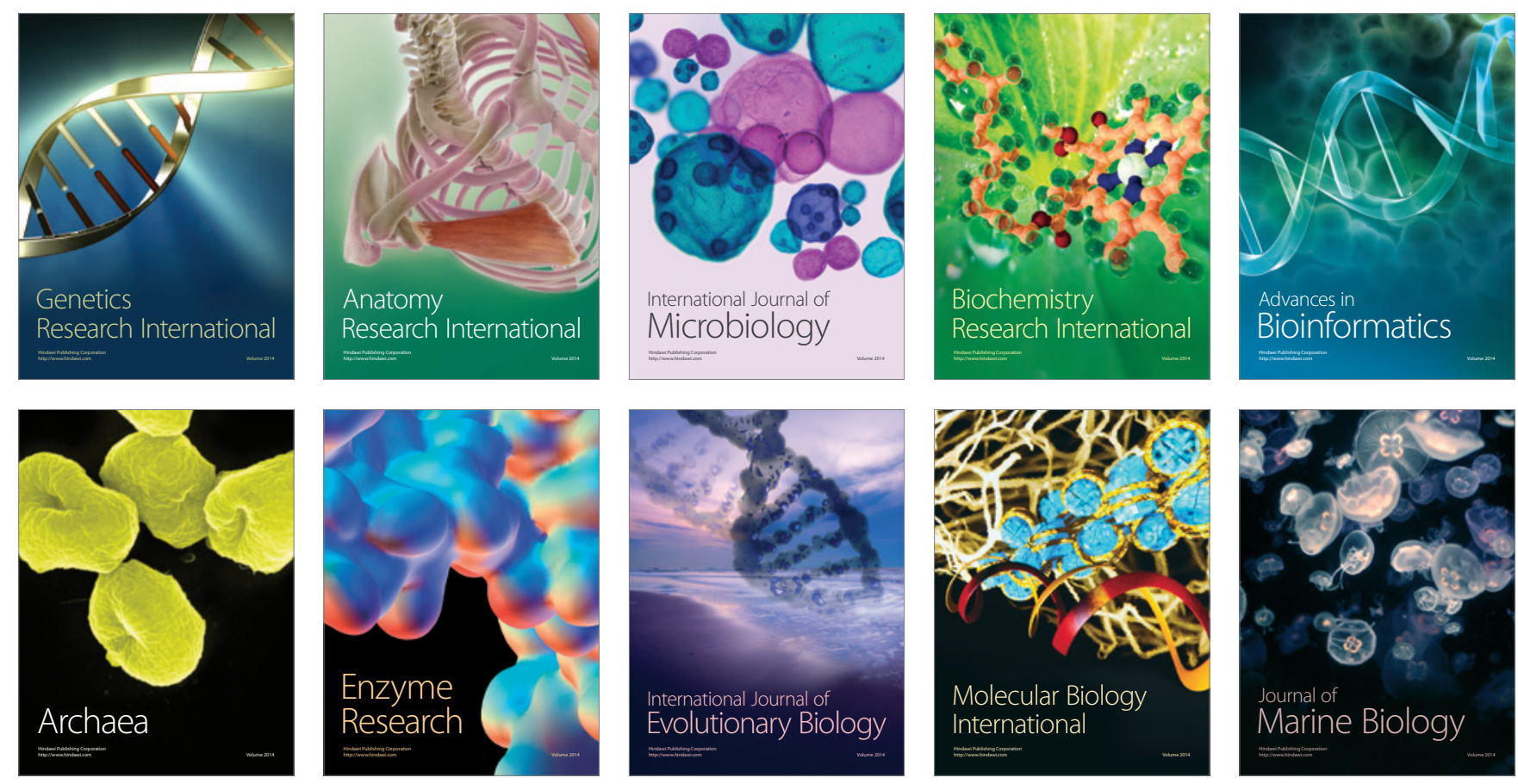